\title{
Reliability analysis of beams on random elastic foundations
}

\author{
D. V. GRIFFITHS*,†, J. PAIBOON*, J. HUANG $\dagger$ and G. A. FENTON $\ddagger, \S$
}

\begin{abstract}
The classical problem of a beam on an elastic foundation has long been of practical interest to geotechnical engineers, because it provides a framework for computing deflections not only of foundations, but also of vertically oriented laterally loaded piles. The supporting soil can be modelled as an elastic medium, which can be calibrated to represent the stiffness of the soils adjacent to the beam (or pile). The objective of this paper is to study the influence of spatially random soil stiffness on deformations of transversely loaded homogeneous piles and beams, using a combination of finiteelement analysis, random field theory and Monte Carlo simulations. Following code validation against alternative solutions, the method investigates how the statistically defined soil stiffness (mean, standard deviation and spatial correlation length) influences the mean and standard deviation of pile or beam deflection. The goal of such an approach is to estimate the probability of deflections exceeding some design threshold.
\end{abstract}

KEYWORDS: deformation; elasticity; finite-element modelling; footings/foundations; piles

\section{INTRODUCTION}

Analysis of a beam on an elastic foundation is a classical problem first introduced by Winkler in the nineteenth century and later developed by many other investigators, most notably by Hetenyi (1946). These models represent the soil stiffness perpendicular to the axis of the beam by a single coefficient of subgrade reaction, $k$, which may be defined as the ratio of the load per unit length to the local deflection (e.g. Randolph, 1981). Commonly used solutions to this system assume that the coefficient of subgrade reaction is constant or linearly increasing with depth. In this paper, however, the response of a laterally loaded beam or pile on soil with spatially random stiffness is considered. Related work has been reported using stochastic finite-element methods (e.g. Ramu \& Ganesar, 1995; Zhang \& Ellingwood, $1995)$ and using two-dimensional finite elements with random fields (Haldar \& Basu, 2011). In this study the authors expand on previous work (Griffiths et al., 2008; Paiboon, 2008) using the random finite-element method (RFEM), which accounts fully for spatial variability and local averaging. In this method, conventional finite-element analysis of a slender beam on a one-parameter elastic foundation (e.g. Smith \& Griffiths, 2004) is combined with random field generation (e.g. Fenton \& Vanmarcke, 1990) and Monte Carlo simulations to develop output statistics for quantities of interest, such as beam or pile deflection. The use of linear elastic analysis in geotechnical engineering, especially in the prediction of deformations at working stress levels, remains a widely accepted model, provided the modulus is fitted appropriately from laboratory test data, and at representative strain and stress levels (e.g. Poulos \& Davis, 1974,

Manuscript received 19 October 2011; revised manuscript accepted 28 May 2012. Published online ahead of print 16 October 2012.

Discussion on this paper closes on 1 July 2013, for further details see p. ii.

* Department of Civil and Environmental Engineering, Colorado School of Mines, Golden, USA.

$\dagger$ Australian Research Council Centre of Excellence for Geotechnical Science and Engineering, University of Newcastle, Callaghan, Australia.

$\ddagger$ Department of Engineering Mathematics, Dalhousie University, Halifax, Canada.

$\S$ Faculty of Civil Engineering and Geosciences, Delft University of Technology, The Netherlands.
1980; Poulos, 1989; Clayton, 2011).

The goal of the investigations described in this paper is to obtain a probability of design failure or inadequate performance, as opposed to the conventional working stress approach, which leads to a factor of safety. For example, in the analysis of a laterally loaded pile in a spatially random soil, we might be interested in estimating the probability of the top deflection exceeding some allowable design value. In a foundation, we might be interested in the probability that the differential settlement will exceed some acceptable level. As will be shown, these design outcomes can be quantified by counting realisations that give excessive deflections, or by fitting a probability density function to the output.

The paper considers two examples: first, a laterally loaded pile (Prakash \& Sharma, 1990; Reese \& Van Impe, 2001) involving a soil of constant mean stiffness; and second, a beam on a foundation of linearly varying mean stiffness (Hetenyi, 1946).

\section{REVIEW OF ANALYTICAL SOLUTIONS Uniform $k$}

The governing equation of a beam on a uniform elastic foundation supporting a distributed load $q$ is

$$
E I \frac{\mathrm{d}^{4} y}{\mathrm{~d} x^{4}}+k y=q
$$

where $E I$ is the flexural stiffness of the beam, $k$ is the lateral soil stiffness, and $y$ is the transverse deflection at $x$.

From Fig. 1(a), the equation for the lateral deflection $y$ at any point $x$ along a vertical pile of length $L$ and flexural stiffness $E I$ embedded in a soil of constant lateral stiffness $k$ subjected to a lateral top load $P$ is given as (Hetenyi, 1946) where

$$
\begin{aligned}
& y=\frac{2 P \lambda}{k}\left(\frac{A_{1}-A_{2}}{A_{3}}\right) \\
& A_{1}=\sinh (\lambda L) \cos (\lambda x) \cosh [\lambda(L-x)] \\
& A_{2}=\sin (\lambda L) \cosh (\lambda x) \cos [\lambda(L-x)] \\
& A_{3}=\sinh ^{2}(\lambda L)-\sin ^{2}(\lambda L)
\end{aligned}
$$

The relative stiffness $\lambda$ between the pile and the surrounding soil is given as 


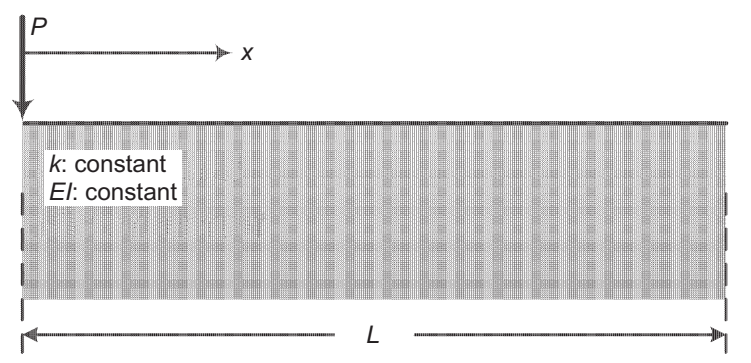

(a)

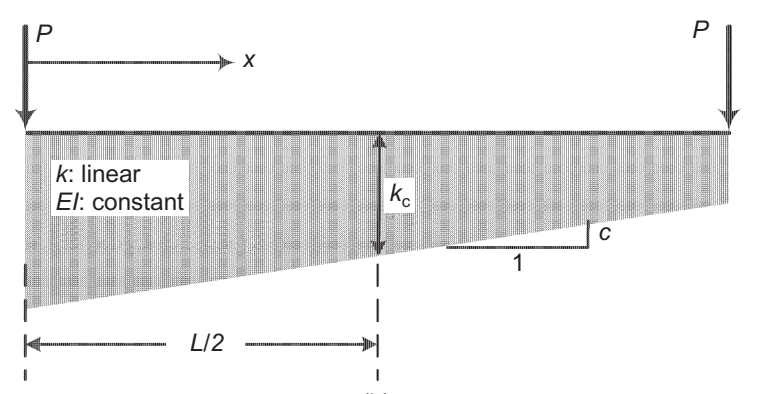

(b)

Fig. 1. Beam on an elastic foundation with (a) constant and (b) linear stiffness

$$
\lambda=\sqrt[4]{\frac{k}{4 E I}}
$$

Hetenyi defined the dimensionless characteristic length of the pile as $\lambda L$, where $\lambda L \leqslant \pi / 4$ is a 'short beam', $\pi / 4<\lambda L \leqslant \pi$ is a 'medium-length beam', and $\lambda L>\pi$ is a 'long beam'. A long beam deforms with predominantly bending deformations, whereas a short beam deforms as an almost rigid body.

\section{Linearly varying $k$}

For the case of a linearly varying $k$, as shown in Fig. 1(b), the present authors have modified Hetenyi's (1946) formulation by making the mid-point stiffness $k_{\mathrm{C}}$ the reference value rather than the end value, thereby avoiding the possibility of dividing by zero. The linear stiffness function with gradient $c$ can then be written as

$$
k=k_{\mathrm{C}}-\frac{1}{2}(L-2 x) c
$$

and the governing equation from equation (1) becomes

$$
E I \frac{\mathrm{d}^{4} y}{\mathrm{~d} x^{4}}+\left[k_{\mathrm{C}}-\frac{1}{2}(L-2 x) c\right] y=q
$$

For point loading as in Fig. 1, where $q=0$ in the majority of elements, equation (5) can be divided by $k_{\mathrm{C}}$ to give

$$
\frac{\mathrm{d}^{4} y}{\mathrm{~d} \xi^{4}}+\alpha \xi y=0
$$

where

$$
\xi=\frac{2 k_{\mathrm{C}}-(L-2 x) c}{2 k_{\mathrm{C}}}
$$

and

$$
\alpha=\frac{k_{\mathrm{C}}^{5}}{c^{4} E I}
$$

Continuing with Hetenyi's formulation, the general solution to equation (6) can be written as

$$
y=C_{1} y_{1}+C_{2} y_{2}+C_{3} y_{3}+C_{4} y_{4}
$$

where

$$
\begin{aligned}
y_{1}= & -\frac{\alpha}{5 !} \xi^{5}+\frac{6 \alpha^{2}}{10 !} \xi^{10}-\frac{6 \times 11 \alpha^{3}}{15 !} \xi^{15} \\
& +\frac{6 \times 11 \times 16 \alpha^{4}}{20 !} \xi^{20}-\ldots \\
y_{2}= & \xi-\frac{2 \alpha}{6 !} \xi^{6}+\frac{2 \times 7 \alpha^{2}}{11 !} \xi^{11}-\frac{2 \times 7 \times 12 \alpha^{3}}{16 !} \xi^{16} \\
& +\frac{2 \times 7 \times 12 \times 17 \alpha^{4}}{21 !} \xi^{21}-\ldots \\
y_{3}= & \frac{\xi^{2}}{2 !}-\frac{3 \alpha}{7 !} \xi^{7}+\frac{3 \times 8 \alpha^{2}}{12 !} \xi^{12}-\frac{3 \times 8 \times 13 \alpha^{3}}{17 !} \xi^{17} \\
& +\frac{3 \times 8 \times 13 \times 18 \alpha^{4}}{22 !} \xi^{22}-\ldots \\
y_{4}= & \frac{\xi^{3}}{3 !}-\frac{4 \alpha}{8 !} \xi^{8}+\frac{4 \times 9 \alpha^{2}}{13 !} \xi^{13}-\frac{4 \times 9 \times 14 \alpha^{3}}{18 !} \xi^{18} \\
& +\frac{4 \times 9 \times 14 \times 19 \alpha^{4}}{23 !} \xi^{23}-\ldots
\end{aligned}
$$

Noting from equation (7) that

$$
\frac{\mathrm{d} \xi}{\mathrm{d} x}=\frac{c}{k_{\mathrm{C}}}
$$

it is possible to obtain, by successive differentiation of equation (9), the angular rotation $\theta$, bending moment $M$ and shearing force $Q$ at any point on the beam as

$$
\begin{aligned}
\theta & =\frac{\mathrm{d} y}{\mathrm{~d} x}=\frac{\mathrm{d} y}{\mathrm{~d} \xi}\left(\frac{\mathrm{d} \xi}{\mathrm{d} x}\right)=\frac{c}{k_{\mathrm{C}}} \frac{\mathrm{d} y}{\mathrm{~d} \xi} \\
M & =-E I \frac{\mathrm{d}^{2} y}{\mathrm{~d} x^{2}}=-E I \frac{\mathrm{d}^{2} y}{\mathrm{~d} \xi^{2}}\left(\frac{\mathrm{d} \xi}{\mathrm{d} x}\right)^{2} \\
& =-E I\left(\frac{c}{k_{\mathrm{C}}}\right)^{2} \frac{\mathrm{d}^{2} y}{\mathrm{~d} \xi^{2}} \\
Q & =-E I \frac{\mathrm{d}^{3} y}{\mathrm{~d} x^{3}}=-E I \frac{\mathrm{d}^{3} y}{\mathrm{~d} \xi^{3}}\left(\frac{\mathrm{d} \xi}{\mathrm{d} x}\right)^{3} \\
& =-E I\left(\frac{c}{k_{\mathrm{C}}}\right)^{3} \frac{\mathrm{d}^{3} y}{\mathrm{~d}^{3}}
\end{aligned}
$$

The known boundary conditions are the shear force $Q$ and bending moment $M$ at each end of the beam, which for the case shown in Fig. 1(b) are

$$
\begin{array}{ll}
M_{x=0}=0, & M_{x=L}=0 \\
Q_{x=0}=-P & Q_{x=L}=P
\end{array}
$$

leading to four linear simultaneous equations in the unknown $C_{i}, i=1,2,3,4$, as

$$
\begin{aligned}
& \left.\frac{\mathrm{d}^{2} y}{\mathrm{~d} \xi^{2}}\right|_{x=0}=0 \\
& \left.\frac{\mathrm{d}^{2} y}{\mathrm{~d} \xi^{2}}\right|_{x=L}=0
\end{aligned}
$$




$$
\begin{aligned}
& \left.\frac{\mathrm{d}^{3} y}{\mathrm{~d} \xi^{3}}\right|_{x=0}=\frac{P}{E I}\left(\frac{k_{\mathrm{C}}}{c}\right)^{3} \\
& \left.\frac{\mathrm{d}^{3} y}{\mathrm{~d} \xi^{3}}\right|_{x=L}=-\frac{P}{E I}\left(\frac{k_{\mathrm{C}}}{c}\right)^{3}
\end{aligned}
$$

\section{REVIEW OF FINITE-ELEMENT SOLUTION}

While the finite-difference method has sometimes been the preferred numerical solution technique for equation (1), this paper uses the finite-element approach, which offers a convenient vehicle for dealing with boundary conditions and variable material properties, especially the random properties described later in the paper. As shown in Fig. 2, a classical slender beam element of stiffness $E I$ is combined with a foundation of stiffness $k$ to give a modified 'BOEF element' (e.g. Smith \& Griffiths, 2004).

The structural slender beam element stiffness matrix

$$
[\mathbf{k}]=\frac{2 E I}{L^{3}}\left[\begin{array}{cccc}
6 & 3 L & -6 & 3 L \\
3 L & 2 L^{2} & -3 L & L^{2} \\
-6 & -3 L & 6 & -3 L \\
3 L & L^{2} & -3 L & 2 L^{2}
\end{array}\right]
$$

is augmented by the stiffness matrix due to the foundation (essentially the beam 'mass' matrix)

$$
[\mathbf{m}]=\frac{k L}{420}\left[\begin{array}{cccc}
156 & 22 L & 54 & -13 L \\
22 L & 4 L^{2} & 13 L & -3 L^{2} \\
54 & 13 L & 156 & -22 L \\
-13 L & -3 L^{2} & -22 L & 4 L^{2}
\end{array}\right]
$$

to give the combined stiffness of the BOEF element as

$$
\left[\mathbf{k}^{\prime}\right]=[\mathbf{k}]+[\mathbf{m}]
$$

This is followed by assembly, implementation of boundary conditions, introduction of loads and equation solution. Later in this paper, the stiffness parameter $k$ in equation (16) will be treated as a random variable.

\section{EXAMPLE PROBLEMS}

Example 1: Laterally loaded pile in a soil of uniform stiffness

The first example is taken from Prakash \& Sharma (1990), and is of the type shown in Fig. 1(a). A pile of length $L=12 \cdot 2 \mathrm{~m}$ and stiffness $E I=9492 \mathrm{kN} \mathrm{m}^{2}$ is driven into clay and subjected to a lateral top load of $P=28 \mathrm{kN}$. The lateral soil stiffness $k$ is constant, and given by

Beam stiffness, El: $\mathrm{kN} \mathrm{m}^{2}$

Foundation stiffness, $k: \mathrm{kN} / \mathrm{m}^{2}$

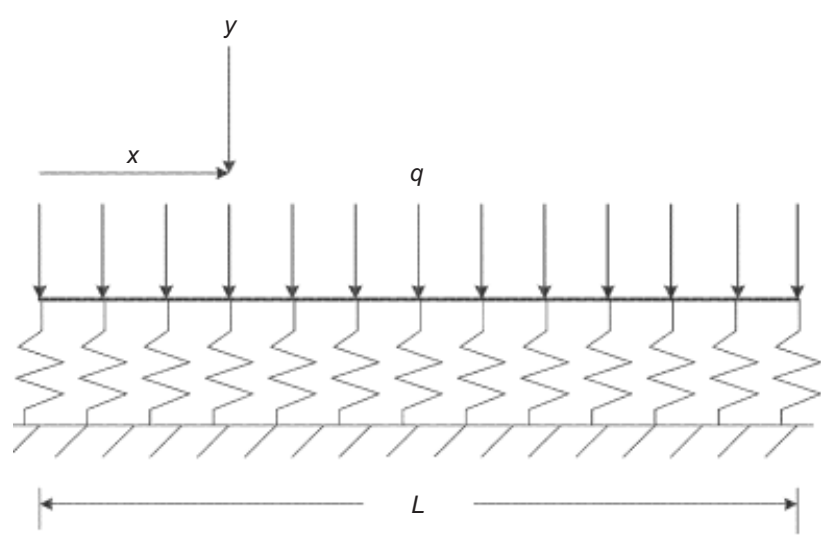

Fig. 2. Beam on an elastic foundation element $k=5774 \mathrm{kPa}$. From equation (3), $\lambda L=7 \cdot 619$ : hence the pile can be considered 'long'.

The analytical solution of the deflection at the top $(x=0)$ for this case from equation (2) is given as $y=6.1 \mathrm{~mm}$, which is compared with finite-element analysis using the published software of Smith \& Griffiths (2004) (program p43) using two, four and eight equal-length beam elements in Table 1. Good agreement is obtained using even the coarsest finite-element mesh. Fig. 3 shows the deflected shape as computed by the two methods over the entire length of the pile.

\section{Example 2: Foundation on a linearly varying foundation}

The second example is adapted from Hetenyi (1946), and is of the type shown in Fig. 1(b). A beam of length $L=3.048 \mathrm{~m}$ and stiffness $E I=1033 \mathrm{kN} \mathrm{m}^{2}$ is subjected to equal point loads at each end of $P=20 \mathrm{kN}$. The underlying foundation stiffness $k$ decreases linearly from $k=4826 \mathrm{kPa}$ at the left end $(x=0)$ to $k=689 \mathrm{kPa}$ at the right end $(x=L)$ : hence $k_{\mathrm{C}}=2758 \mathrm{kPa}$ and $c=-1357 \mathrm{kPa} / \mathrm{m}$, giving $\alpha=45.56$ from equation (8). From equation (3), by letting $k=k_{\mathrm{C}}$ it is seen that $\lambda L=2.755$ : hence the beam can be considered medium length.

The analytical solution for this case from equations (4)(14) gives

$$
y=0.0299 y_{1}-0.0300 y_{2}-0.0463 y_{3}+0.1973 y_{4}
$$

The end deflections are obtained by substitution of the dimensionless variable $\xi=1.7498$ and $\xi=0.2501$ (corresponding to $x=0$ and $x=L=3.048 \mathrm{~m}$ respectively) into equations (10) to give $y_{1}, y_{2}, y_{3}$ and $y_{4}$, which in turn are substituted into equation (18) to give the results shown in the analytical column of Table 2 . Substitution of a full range of $1.7498 \leqslant \xi \leqslant 0.2501$ into equations (10) gives the analy-

Table 1. Top deflection $(x=0)$ by finite-element and analytical solutions (mm)

\begin{tabular}{l|c|c|c}
\hline \multirow{2}{*}{ Analytical (equation (2)) } & \multicolumn{3}{|c}{ FE solution: number of elements } \\
\cline { 2 - 4 } & 2 & 4 & 8 \\
\hline $6 \cdot 1$ & $5 \cdot 8$ & $5 \cdot 9$ & $6 \cdot 0$ \\
\hline
\end{tabular}

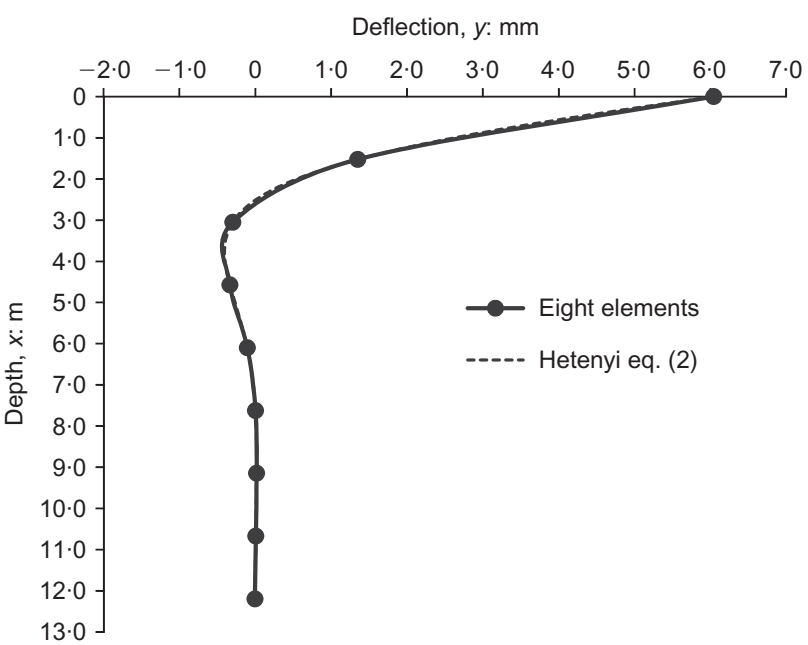

Fig. 3. Deflected shape of Example 1 pile from finite-element and analytical solutions 
Table 2. Beam end deflections $(x=0, x=L)$ by finite-element and analytical solutions (mm)

\begin{tabular}{l|c|c|c|c|c|c|c}
\hline \multicolumn{2}{l|}{$\begin{array}{l}\text { Analytical (equations } \\
\text { (4)-(14), (18)) }\end{array}$} & \multicolumn{6}{|c}{ FE solution: number of elements } \\
\cline { 3 - 8 } \multicolumn{2}{l|}{} & \multicolumn{2}{|c|}{2} & \multicolumn{2}{|c}{4} & \multicolumn{2}{c}{8} \\
\hline$x=0$ & $x=L$ & $x=0$ & $x=L$ & $x=0$ & $x=L$ & $x=0$ & $x=L$ \\
\hline 6.6 & 21.4 & 8.0 & $16 \cdot 2$ & $7 \cdot 0$ & $19 \cdot 8$ & $6 \cdot 7$ & $21 \cdot 0$ \\
\hline
\end{tabular}

tical deflection profile shown compared with an eight-finiteelement solution in Fig. 4.

\section{PROBABILISTIC ANALYSIS}

In this section the stiffness of the soil $k$ is considered to be a random variable. It is then possible to examine the influence of the mean and standard deviation of $k\left(\mu_{k}, \sigma_{k}\right)$ on the mean and standard deviation of the pile-top deflection in the first test problem, and the mean and standard deviation of the (differential) deflection at each ends of the beam in the second test problem. In the more advanced analyses using random fields, the spatial correlation length $\theta_{k}$ (or $\left.\theta_{\ln k}\right)$ and the probability density function of the random foundation stiffness $k$ are also considered.

Initially, the pile in the first test problem is analysed using a simplified approach called the first-order second-moment (FOSM) method.

Consider a nonlinear function $Y$ of a single random variable $X$

$$
Y=f(X)
$$

where the mean and standard deviation of $X$ are given as $\mu_{X}$ and $\sigma_{X}$ respectively. The mean and standard deviation of the function $Y$ by FOSM are then

$$
\begin{gathered}
\mu_{Y} \approx f\left(\mu_{X}\right) \\
\sigma_{Y} \approx \frac{\mathrm{d} f}{\mathrm{~d} x} \sigma_{X}
\end{gathered}
$$

where the derivative term is evaluated at the mean, $x=\mu_{X}$.

Returning to test example 1 , consider a simplified version of Hetenyi's formula from equation (2) for the pile top $(x=0)$ in which the quotient involving trigonometric and hyperbolic functions is treated as a constant evaluated at the mean $k=\mu_{k}=5774 \mathrm{kN} / \mathrm{m}^{2}$. With other quantities fixed to

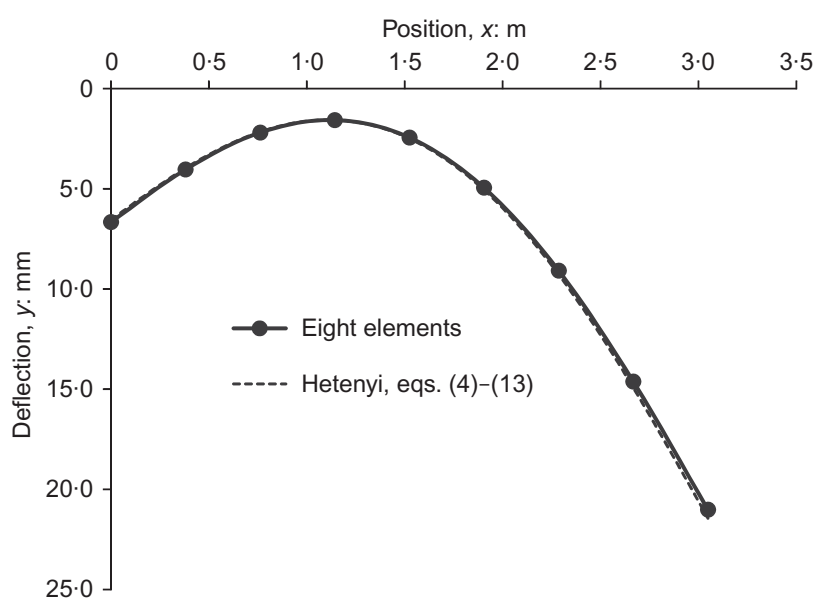

Fig. 4. Deflected shape of Example 2 beam from finite-element and analytical solutions
$L=12 \cdot 2 \mathrm{~m}$ and $E I=9492 \mathrm{kNm}^{2}$, as used by Prakash \& Sharma (1990), the following is obtained

$$
\frac{\sinh (\lambda L) \cosh (\lambda L)-\sin (\lambda L) \cos (\lambda L)}{\sinh ^{2}(\lambda L)-\sin ^{2}(\lambda L)}=1.0000
$$

Further inspection of this quotient reveals that it is remarkably constant for all reasonable values of $k$.

From equation (2), and noting the definition of $\lambda$ from equation (3),

$$
y=\frac{2 P \lambda}{k}=\frac{1}{k^{3 / 4}} \frac{\sqrt{2} P}{(E I)^{1 / 4}}
$$

which after substitution of $P=28 \mathrm{kN}$ and $E I=9492 \mathrm{kN} \mathrm{m}^{2}$ gives

$$
y=\frac{4 \cdot 0118}{k^{3 / 4}}
$$

Taking logs of both sides of equation (24) gives

$$
\begin{aligned}
\ln y & =\ln 4.0118-0.75 \ln k \\
& =1.3892-0.75 \ln k
\end{aligned}
$$

If $\ln k$ and $\ln y$ are now treated as random variables in equation (25), and noting there is a linear function (of the $\ln k$ terms), FOSM will be exact, and application of equations (20)-(21) gives

$$
\begin{aligned}
\mu_{\ln y} & =1.3892-0.75 \mu_{\ln k} \\
\sigma_{\ln y} & =0.75 \sigma_{\ln k}
\end{aligned}
$$

If $k$ is log-normal, and for now it is assumed that its coefficient of variation $V_{k}=\sigma_{k} / \mu_{k}=1 \cdot 0$ (i.e. $\sigma_{k}=\mu_{k}=$ $5774 \mathrm{kPa}$ ), the properties of the underlying normal distribution of $\ln k$ are given by the transformation equations

$$
\begin{aligned}
& \sigma_{\ln k}=\sqrt{\ln \left(1+V_{k}^{2}\right)}=0.8326 \\
& \mu_{\ln k}=\ln \mu_{k}-\frac{1}{2} \sigma_{\ln k}^{2}=8.3145
\end{aligned}
$$

Hence from equations (26)-(27)

$$
\begin{aligned}
& \mu_{\ln y}=-4.8467 \\
& \sigma_{\ln y}=0.6244
\end{aligned}
$$

Assuming $\ln y$ is normal, it is possible to transform back to the log-normal variable $y$ using the inverse of equations (28) $-(29)$ to give

$$
\begin{aligned}
& \mu_{y}=\exp \left(\mu_{\ln y}+\frac{1}{2} \sigma_{\ln y}^{2}\right)=0.0095 \mathrm{~m} \\
& \sigma_{y}=\mu_{y} \sqrt{\exp \left(\sigma_{\ln y}^{2}\right)-1}=0.0066 \mathrm{~m}
\end{aligned}
$$

Example 2 is less amenable to analysis using a simplified method such as FOSM, since the underlying foundation has 
a random stiffness $k$ that varies linearly. This type of problem needs a more advanced approach, such as the RFEM described in the next section.

\section{CHOICE OF LOG-NORMAL}

It is known that stiffness cannot be negative, and has no arbitrary upper bound. This means that its distribution must be non-negative, and skewed to the right. There are a number of common distributions having these features, including gamma, Weibull, Rayleigh, chi-square and log-normal, but the authors are not aware of any statistical studies promoting one of these distributions over the others for representing soil stiffness. In any case, the shapes of these distributions are all very similar, once the first two moments are specified, and it is not expected that any of them will lead to significantly different results. The log-normal distribution was selected for the studies presented in this paper, because of its simple relationship with the normal distribution, whose joint distribution is entirely specified by the first two moments (mean and variance).

\section{RANDOM FIELDS}

In this section, spatially random soil adjacent to the pile is introduced. This is intended to model highly variable soil typical of some sites in which the soil stiffness is characterised by a mean, a standard deviation and a spatial correlation length. The RFEM developed by Griffiths \& Fenton in the early 1990 s combines finite-element analysis with random field theory (Fenton \& Vanmarcke, 1990) in conjunction with Monte Carlo simulations. The methodology has been applied successfully to several areas of geotechnical engineering, and the interested reader is referred to Fenton \& Griffiths (2008) for greater detail of the methodology.

In this study, the pile is divided into 100 elements of equal length, and a log-normal distribution of foundation stiffness is mapped onto each element. The log-normal random variable is defined by three parameters: the mean $\left(\mu_{k}\right)$, the standard deviation $\left(\sigma_{k}\right)$ and the spatial correlation length $\left(\theta_{\ln k}\right)$. A log-normally distributed random variable $k$ has a normally distributed $\ln k$ : thus the random field of $k$ at any particular location $x_{i}$ is generated using the transformation

$$
k\left(x_{i}\right)=\exp \left[\mu_{\ln k}+\sigma_{\ln k} G_{\mathrm{n}}\left(x_{i}\right)\right]
$$

$G_{\mathrm{n}}\left(x_{i}\right)$ is a standard normal distribution with zero mean, unit variance and spatial correlation function $\rho(\tau)$ defined by

$$
\rho(\tau)=\exp \left\{\frac{-2|\tau|}{\theta_{\ln k}}\right\}
$$

where $|\tau|$ is the absolute distance between any two points, and $\theta_{\ln k}$ is the spatial correlation length in $\log$ space. The spatial correlation length can be thought of as the distance (in length units) over which properties are reasonably well correlated. For example, two points in a one-dimensional random field separated by $\theta_{\ln k}$ will have an average correlation from equation (35) of about $\rho\left(\theta_{\ln k}\right) \approx 0 \cdot 14$.

From equation (34), each element is assigned a $k$ value that varies from one element to the next. A small spatial correlation length implies rapidly varying properties, whereas a large spatial correlation length implies gradually varying properties. Two random fields with the same mean and standard deviation could have quite different spatial correlation lengths. In the current work the spatial correlation length has been expressed in dimensionless form as

$$
\Theta_{\ln k}=\frac{\theta_{\ln k}}{L}
$$

where $L$ is the total beam length.

Figure 5 shows a typical random field realisation of foundation stiffness, where the dark and light regions depict, respectively, stiff and less stiff soil.

The choice of soil parameters for probabilistic geotechnical analysis (e.g. mean, standard deviation and spatial correlation length) is generally site specific and often challenging, owing to insufficient site data. There may be the additional issue of anisotropy, since many sedimentary deposits exhibit a longer spatial correlation length in the horizontal direction than in the vertical. Boreholes are usually vertical, so more is typically known about the vertical correlation length (e.g. Fenton, 1999). There is an increasing bibliography, however, recognising the importance of spatial correlation in probabilistic geotechnical analysis, which provides typical ranges of probabilistic soil properties and spatial correlation lengths (e.g. Lee et al., 1983; Phoon \& Kulhawy, 1995; Duncan, 2000). For design purposes, however, and in the absence of good spatial correlation data, the focus shifts to parametric studies, and to an investigation of the worst-case spatial correlation lengths that lead to the highest probability of design failure and inadequate performance.

\section{VARIANCE REDUCTION OVER A ONE-DIMENSIONAL BEAM ELEMENT}

The input statistics $\left(\mu_{k}, \sigma_{k}\right)$ are assumed to be provided at the 'point' resolution, but each element is given a single value of the random variable: thus local averaging must be accounted for. The RFEM takes account of element size in the random field generation, and delivers statistically consistent values of the locally averaged properties. For a single one-dimensional beam finite element of length $l=\alpha_{l} \theta_{\ln k}$, where $\alpha_{l}$ is a dimensionless length parameter, and a correlation function given by equation (35), it can be shown (e.g. Vanmarcke, 1984) that the local averaging variance reduction factor for a normal variant is given by

$$
\gamma=\frac{2}{\left(\alpha_{l} \theta_{\ln k}\right)^{2}} \int_{0}^{\alpha_{l} \theta_{\ln k}}\left(\alpha_{l} \theta_{\ln k}-x\right) \exp \left\{-2 x / \theta_{\ln k}\right\} \mathrm{d} x
$$

where $\gamma=\sigma_{k(A)}^{2} / \sigma_{k}^{2}$, and $\sigma_{k(A)}^{2}$ is the variance after local averaging. For the one-dimensional case, the variance reduction factor from equation (37) can be evaluated analytically to give

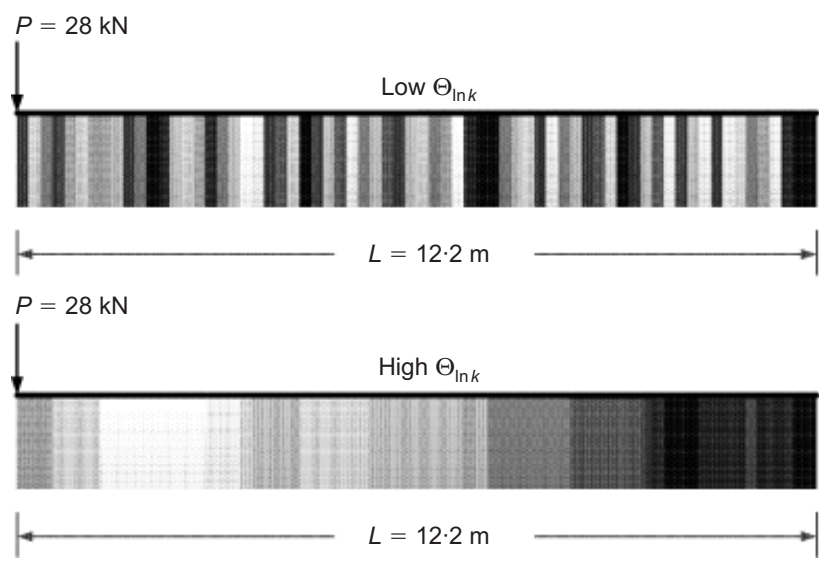

Fig. 5. Typical random fields of foundation stiffness mapped onto a mesh of 100 elements in Example 1 


$$
\gamma=\frac{1}{2}\left(\frac{2 \alpha_{l}+\mathrm{e}^{-2 \alpha_{l}}-1}{\alpha_{l}^{2}}\right)
$$

It may be noted that arithmetic averaging of $\ln k$ applied using equation (38) preserves the median of a log-normal distribution of $k$, but causes both its mean and its standard deviation to fall: thus $\mu_{k(A)}<\mu_{k}$ and $\sigma_{k(A)}<\sigma_{k}$. The laterally loaded pile model in Fig. 5 with 100 elements $(l=0.122 \mathrm{~m})$ and a low spatial correlation length of $\theta_{\ln k}=1 \mathrm{~m}\left(\alpha_{l}=0 \cdot 122\right)$ leads to a variance reduction factor from equation (38) of $\gamma=0.9234$. A longer spatial correlation length of $\theta_{\ln k}=5 \mathrm{~m} \quad\left(\alpha_{l}=0.0244\right)$ would give $\gamma=0.9839$.

As an example, a log-normal variant with $\mu_{k}=\sigma_{k}=$ $5774 \mathrm{kPa}$ has underlying normal properties from equations (28)-(29) of $\sigma_{\ln k}=0.8326$ and $\mu_{\ln k}=8 \cdot 3145$. Arithmetic local averaging of $\ln k$ has no influence on $\mu_{\ln k}$, but for the low spatial correlation length of $\theta_{\ln k}=1 \mathrm{~m}$ mentioned above, $\gamma=0.9234$ and $\sigma_{\ln k(A)}=0.8326 \sqrt{0.9234}=0.8001$. Finally, from the inverse equations (32)-(33) the locally averaged parameters of the log-normal distribution become $\mu_{k(A)}=5623 \mathrm{kPa}$ and $\sigma_{k(A)}=5324 \mathrm{kPa}$.

\section{RESULTS OF RFEM ANALYSES}

RFEM analysis of the BOEF problem involves generation of a log-normal random field of foundation stiffness values with user-controlled values of the mean $\left(\mu_{k}\right)$, standard deviation $\left(\sigma_{k}\right)$ and spatial correlation length $\left(\theta_{\ln k}\right)$, as described previously. These values are then mapped onto the finite-element mesh, and a conventional deterministic BOEF analysis performed to compute output quantities of interest such as the end displacement. The process is then repeated; a new random field is generated and a different displacement is computed. While each new random field simulation in the Monte Carlo process has the same underlying statistics, the stiffer and less stiff parts of the foundation field occur in different places, as shown in Fig. 5. For example, if a particular simulation leads to lower stiffness values near the top, then the top displacement would be relatively high. On another simulation, higher stiffness values might occur near the top, in which case the top displacement would be relatively low. After a sufficient number of simulations have been performed, the statistics of the top displacement stabilise and can be interpreted probabilistically, as will be discussed subsequently.

In this paper, only the foundation stiffness given as $k$ in equation (16) is treated as random, so all other quantities such as the beam stiffness, the dimensions and the loading - are held constant.

\section{Example 1: Laterally loaded pile in a random soil}

The results of RFEM analyses with Monte Carlo simulations are presented, based on a range of parametric variations of $V_{k}$ and $\theta_{\ln k}$. A typical realisation of the random foundation field for $V_{k}=0 \cdot 1$ and $\theta_{\ln k}=2 \mathrm{~m}$ is shown in Fig. 6 using 100 elements. In all cases, the mean foundation stiffness was held constant at $\mu_{k}=5774 \mathrm{kPa}$, as shown by the dotted line.

Following each suite of 5000 Monte Carlo simulations, the mean and standard deviation of the pile top deflection $\left(\mu_{y}, \sigma_{y}\right)$ were calculated, and are shown plotted in Figs 7 and 8 .

As observed in other elastic deformation problems with variable stiffness (e.g. Griffiths \& Fenton, 2009), the more flexible parts of the system dominate the solution, and the mean deflection $\mu_{y}$ is higher than the deterministic value

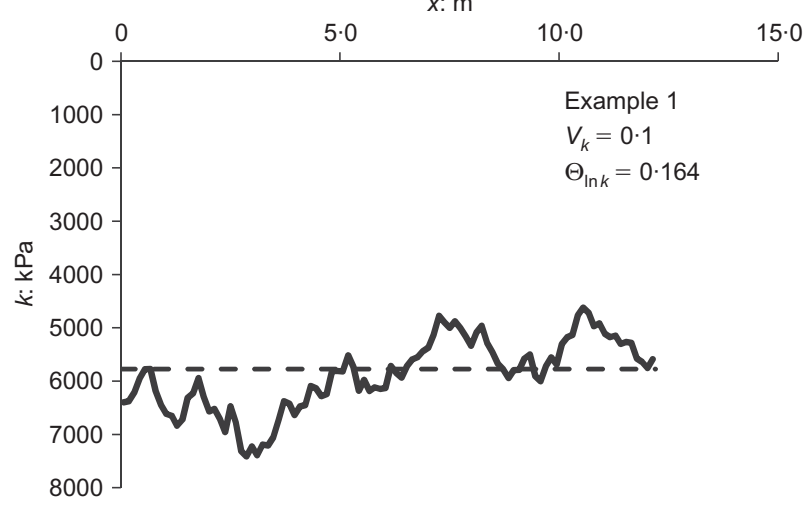

Fig. 6. Typical realisation of a random foundation field in Example 1 with $\mu_{k}=5774 \mathrm{kPa}$

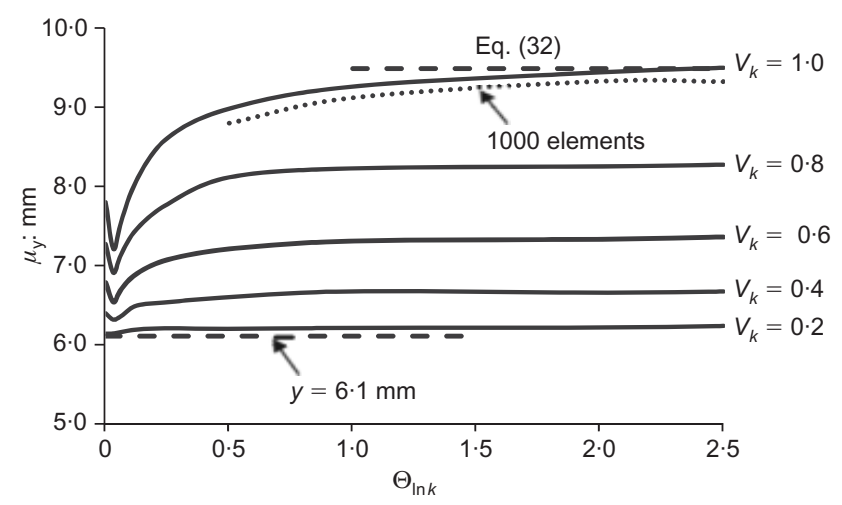

Fig. 7. $\mu_{y}$ against $\Theta_{\ln k}$ for different $V_{k}$ values $\left(\mu_{k}=5774 \mathrm{kPa}\right)$

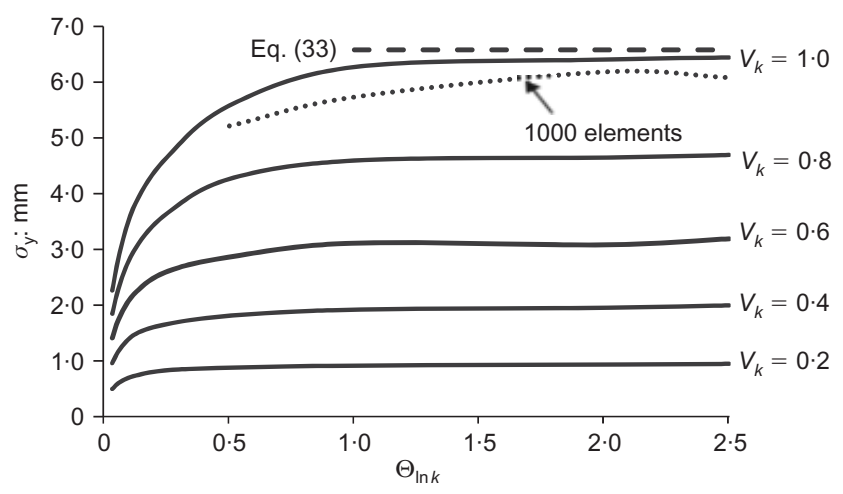

Fig. 8. $\sigma_{y}$ against $\Theta_{\ln k}$ for different $V_{k}$ values $\left(\mu_{k}=5774 \mathrm{kPa}\right)$

based on the mean for all combinations of $V_{k}$ and $\Theta_{\ln k}$, as shown in Fig. 7. With a low $V_{k}$, the mean top deflection is close to the deterministic result of $6 \cdot 1 \mathrm{~mm}$ obtained with a constant stiffness of $k=5774 \mathrm{kPa}$ from Table 1 . For all $V_{k}$, the rate of increase of $\mu_{y}$ slows considerably for $\Theta_{\ln k} \gg 1$, and for the case when $V_{k}=1$ tends to the result obtained using FOSM of $\mu_{y}=0.0095 \mathrm{~m}$ from equation (32).

The results for $\sigma_{y}$ in Fig. 8 give a trend similar to those obtained for $\mu_{y}$, with values increasing for all combinations of $V_{k}$ and $\Theta_{\ln k}$. Limiting cases, as might be expected, are that $\sigma_{y} \rightarrow 0$ as $V_{k} \rightarrow 0$, and for larger values of $\Theta_{\ln k} \sigma_{y}$ tends to the result obtained using FOSM from equation (33) of $\sigma_{y}=0.0066 \mathrm{~m}$ for the case when $V_{k}=1$. These checks confirm that the single random variable approaches are essentially special cases of RFEM when $\Theta_{\ln k} \rightarrow \infty$.

A final comment should be made relating to the limiting case of vanishingly short correlation lengths, when $\Theta_{\ln k} \rightarrow 0$. 
In this case, from equation (38), $\gamma \rightarrow 0, \sigma_{k A} \rightarrow 0, \mu_{k A} \rightarrow$ $\operatorname{Median}_{k}=\mathrm{e}^{\mu_{\ln k}}$, and the foundation becomes essentially homogeneous, with a constant stiffness given by $k=\operatorname{Median}_{k}$. The deterministic result corresponding to $\Theta_{\ln k}=0$ is easily checked. For example, if $\mu_{k}=5774 \mathrm{kPa}$ and $V_{k}=1$, then the Median $_{k}=4083 \mathrm{kPa}$, and the deterministic top deflection will be given from equation (2) as $y=7.8 \mathrm{~mm}$. All the curves in Fig. 7 head toward their respective median values as $\Theta_{\ln k} \rightarrow 0$. The graphs in Fig. 8 have not been produced to the origin to avoid clutter, since as would be expected, in all curves, $\sigma_{y} \rightarrow 0$ as $\Theta_{\ln k} \rightarrow 0$.

When a second set of runs was performed using 1000 elements instead of 100 , both the mean and the standard deviation of the top deflection were lowered slightly for all $\Theta_{\ln k}>0$, as shown in Figs 7 and 8, indicating that the results from the coarser mesh are slightly conservative. It was noted, however, that all results converged on the deterministic result corresponding to the median at $\Theta_{\ln k}=0$. With the more refined mesh, the minima in the mean deflections as shown in Fig. 9 occurred at smaller values of $\Theta_{\ln k}$ and at lower values of $\mu_{y}$. Although the details of the response in this part of the plot exhibit mesh dependence, the qualitative behaviour is believed to be correct: that is, the tip experiences a stiffer response when $\Theta_{\ln k}$ is slightly above zero, owing to the small increase in foundation stiffness and variance. This observation is under further investigation.

From a design viewpoint, however, the mesh-sensitive differences observed in the location of the minima are not of great concern, since the most conservative deflection predictions are observed at higher spatial correlation lengths. As will be discussed in the next section, it is when the mean and standard deviation of deflection are greatest that the probability of design failure is most likely to reach unacceptable levels.

\section{Example 1: Probabilistic interpretation}

Of interest in a laterally loaded pile problem might be (for a given top load) an estimate of the probability that the top deflection exceeds some allowable design value. In order to make probabilistic interpretations from a Monte Carlo analysis, it is possible either to count the number of simulations that exceed the allowable deflection, or make an assumption about the probability density function (pdf) that best fits the output values, and then refer to standard cumulative distribution tables. Since the foundation stiffness values were assumed to be log-normal, it seems reasonable to assume that the pdf of the top deflection is also lognormal. Fig. 10 shows a histogram based on 5000 solutions from the RFEM runs, together with a fitted log-normal function based on the computed values of the mean and standard deviation of $\mu_{y}$ and $\sigma_{y}$. Although objective goodness-of-fit tests can be performed, in the interests of brevity it is noted here that the log-normal fit seems reasonable.

For argument's sake, assume that the design has failed if

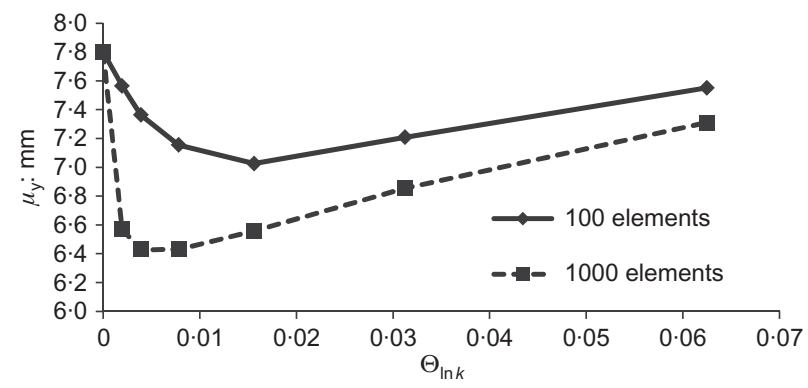

Fig. 9. Influence of mesh density on minima $\left(V_{k}=1\right)$

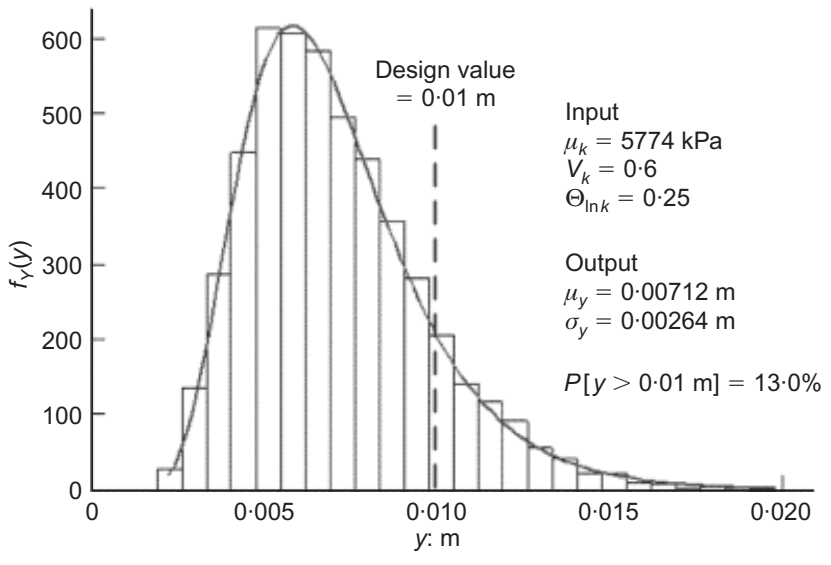

Fig. 10. Histogram and log-normal fit for a typical set of computed deflection values for Example 1

the top deflection exceeds $10 \mathrm{~mm}$. Thus for any particular parametric combination of $V_{k}$ and $\Theta_{\ln k}$, the intention is to estimate $P[y>10 \mathrm{~mm}]$. For the particular case shown in Fig. 10, where $\Theta_{\ln k}=0 \cdot 25$ and $V_{k}=0 \cdot 6$, about 657 out of 5000 simulations led to a top deflection greater than $0.01 \mathrm{~m}$, indicating a probability of failure of about $p_{\mathrm{f}}=0 \cdot 131$. Based on the fitted log-normal distribution, a very similar result can be obtained using the following steps.

\section{Sample calculation.}

(a) For input values $\mu_{k}=5774 \mathrm{kPa}, V_{k}=0.6$ and $\Theta_{\ln k}=$ $0 \cdot 25$.

(b) From Monte Carlo simulations, $\mu_{y}=7.12 \mathrm{~mm}, \sigma_{y}=$ $2.64 \mathrm{~mm}\left(V_{y}=0.37\right.$ log-normal $)$.

(c) Obtain parameters of underlying normal distribution of $\ln y$

$$
\begin{aligned}
& \sigma_{\ln y}=\sqrt{\ln \left(1+V_{y}^{2}\right)}=0.359 \text { and } \\
& \mu_{\ln y}=\ln \mu_{y}-\frac{1}{2} \sigma_{\ln y}^{2}=1.898 \\
& \begin{array}{r}
P[y>10 \mathrm{~mm}]=1-\Phi\left[\frac{\ln 10-\mu_{\ln y}}{\sigma_{\ln y}}\right] \\
=1-\Phi[1.126]=0.130
\end{array}
\end{aligned}
$$

where $\Phi(\cdot)$ is the standard cumulative distribution function.

Figure 11 shows the results of similar probabilistic calcula-

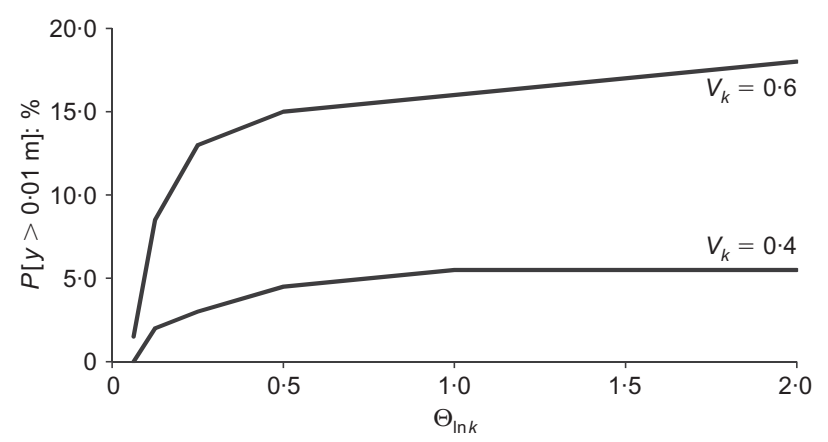

Fig. 11. $\mathrm{P}[y>0.01(\mathrm{~m})]$ against $\Theta_{\ln k}$ for different $V_{k}$ values in Example 1 
tions for other typical values of $V_{k}$ and $\Theta_{\ln k}$. Probabilities of failure corresponding to $V_{k} \leqslant 0 \cdot 2$ were sufficiently small on the scale of Fig. 11 that they are not shown.

\section{Example 2: Beam on a random foundation with linearly} varying mean stiffness

The second example, as shown in Fig. 1(b), consists of a beam on a random foundation with a linearly decreasing mean stiffness. The mean foundation stiffness is given by

$$
\mu_{k}=4826-1357 x \mathrm{kPa}
$$

and in this investigation it is assumed that the coefficient of variation $V_{k}$ is constant: thus the standard deviation also decreases linearly, as given by

$$
\sigma_{k}=V_{k}(4826-1357 x) \mathrm{kPa}
$$

A typical realisation of the random foundation field for $V_{k}=0.1$ and $\theta_{\ln k}=0.5 \mathrm{~m}$ with 100 elements is shown in Fig. 12. In all cases, the linearly varying mean foundation stiffness given by equation (39) was maintained as shown by the dotted line.

A range of parametric variation of $V_{k}$ and $\Theta_{\ln k}$ was considered with 5000 Monte Carlo simulations. To reduce the volume of output, the authors have chosen to present the statistics $\left(\mu_{D}, \sigma_{D}\right)$ of the differential settlement between the ends of the beam following RFEM analysis, where $D=\left|y_{x=L}-y_{x=0}\right|$. The results are plotted in Figs 13 and 14.

As before, it is instructive to consider the limiting values of $\Theta_{\ln k}$. In the case of $\Theta_{\ln k} \rightarrow 0$, the result becomes essentially deterministic, with all stiffness values along the length of the beam tending to their median values. For small values of $V_{k}$ the median is essentially the same as the mean, and $\mu_{D}$ converges on the deterministic value of $14.8 \mathrm{~mm}$, as shown in Table 2. For larger values of $V_{k}$ the median stiffness falls, and both the end and differential settlements tend to larger values as $\Theta_{\ln k} \rightarrow 0$. For example, when

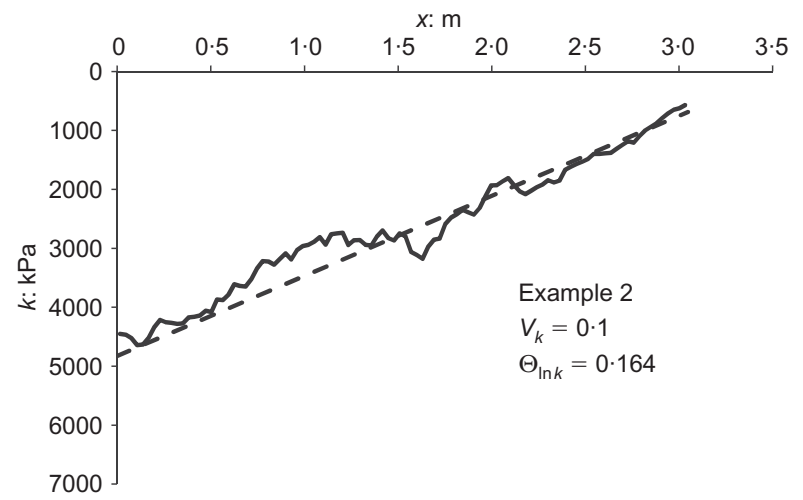

Fig. 12. Typical realisation of a random foundation field in Example 2

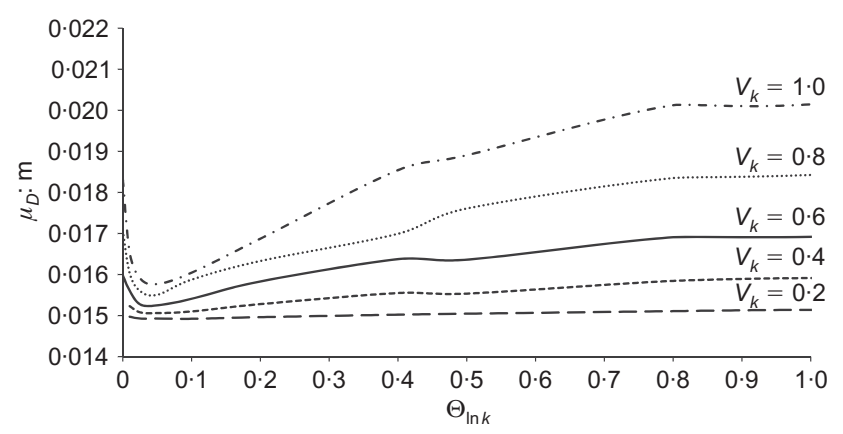

Fig. 13. $\mu_{D}$ against $\Theta_{\ln k}$ for different $V_{k}$ values

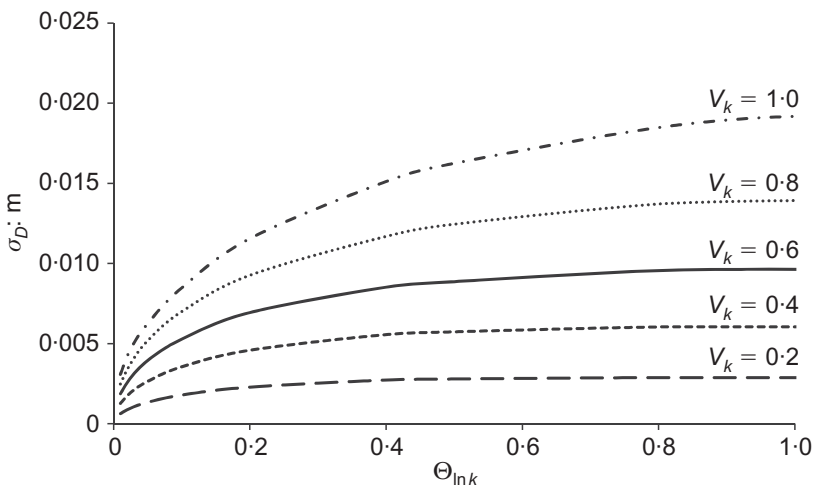

Fig. 14. $\sigma_{D}$ against $\Theta_{\ln k}$ for different $V_{k}$ values

$V_{k}=1$, the mean differential settlement tends to about $18.3 \mathrm{~mm}$. The results for the standard deviation of differential settlement are perhaps as expected, with $\sigma_{D}$ heading to zero as $\Theta_{\ln k} \rightarrow 0$ for all $V_{k}$, and tending to level out at higher values of $\Theta_{\ln k}$.

Figures 13 and 14 both show a generally increasing trend for $\mu_{D}$ and $\sigma_{D}$ as $\Theta_{\ln k}$ increases, with the highest values corresponding to higher values of $V_{k}$. These are similar trends to those shown in the laterally loaded pile example in Figs 7 and 8, which were validated analytically. Similar analytical checks of the asymptotes at high values of $\Theta_{\ln k}$ for this example with linearly varying mean stiffness are the subject of ongoing analysis.

\section{CONCLUDING REMARKS}

The paper has described RFEM analyses of beams on spatially random soil in order to investigate the influence of statistically defined foundation stiffness on beam deflections. The cases considered were: (a) a laterally loaded pile with soil stiffness described by a stationary random field with a constant mean, standard deviation and spatial correlation length; and (b) a beam supported on a soil with a random stiffness defined by a linearly increasing mean, constant coefficient of variation and spatial correlation length. In all cases, the soil stiffness was assumed to be log-normal.

Following deterministic validation against closed-form solutions, a range of parametric studies were conducted with the mean stiffness held constant. By varying the coefficient of variation and spatial correlation length of the soil stiffness, output quantities of interest such as the mean and standard deviation of the pile top deflection, and the beam end (differential) settlements, were computed. With the exception of local averaging effects at small spatial correlation lengths, the results show that increasing the coefficient of variation of the soil stiffness results in an increase in the mean and standard deviation of the pile and beam deflections. Increasing the spatial correlation length of the soil stiffness also results in increased mean and standard deviations of the pile and beam deflections, although this effect levels out when the spatial correlation length approaches the length of the pile or beam. In the case of the laterally loaded pile, a probabilistic interpretation of the computed results was demonstrated, showing how the probability of excessive deflection above some design threshold can be estimated.

The program used in this work, called rBOEF, together with the entire suite of random finite-element programs in source code developed by the authors for geotechnical analysis (e.g. Fenton \& Griffiths, 2008), is available for free download at www.mines.edu/ $\sim$ vgriffit. 


\section{ACKNOWLEDGEMENT}

The authors wish to acknowledge the support of: (a) NSF grant CMMI-0970122 on 'GOALI: Probabilistic geomechanical analysis in the exploitation of unconventional resources'; and (b) KGHM Cuprum, Wrocław, Poland, through the Framework 7 EU project on 'Industrial risk reduction' (IRIS).

\section{NOTATION}

$C_{i}$ equation coefficients $(i=1,2,3,4)$

$c$ stiffness gradient

$D$ differential settlement

EI flexural beam stiffness

$f(\cdot)$ function

$k$ foundation stiffness

[k] element stiffness matrix

$\left[\mathbf{k}^{\prime}\right]$ modified element stiffness matrix

$k_{\mathrm{C}}$ centreline of foundation stiffness

$L$ beam or pile length

$M$ moment

[m] element 'mass' matrix

$P$ point load

$P[\cdot]$ probability

$Q$ shear force

$q$ distributed loading

$V_{k}$ coefficient of variation of $k$

$X$ random variable

$x$ spatial coordinate

$Y$ random variable

$y$ beam or pile transverse displacement

$y_{i} \quad$ solution parameters $(i=1,2,3,4)$

a dimensionless relative beam stiffness parameter

$\alpha_{l}$ dimensionless element size parameter

$\gamma$ variance reduction factor

$\theta$ end rotation

$\theta_{k} \quad$ spatial correlation of $k$

$\theta_{\ln k} \quad$ spatial correlation of $\ln k$

$\lambda$ relative stiffness parameter

$\mu_{D}$ mean of $\mathrm{D}$

$\mu_{k}$ mean of $k$

$\mu_{\ln k}$ mean of $\ln k$

$\mu_{X}$ mean of $X$

$\mu_{Y}$ mean of $Y$

$\xi$ dimensionless beam stiffness

$\rho$ correlation coefficient

$\sigma_{D} \quad$ standard deviation of $D$

$\sigma_{k} \quad$ standard deviation of $k$

$\sigma_{\ln k} \quad$ standard deviation of $\ln k$

$\sigma_{X} \quad$ standard deviation of $X$

$\sigma_{Y} \quad$ standard deviation of $Y$

$\tau$ distance between two points in the random field

\section{REFERENCES}

Clayton, C. R. I. (2011). Stiffness at small strain: research and practice. Géotechnique 61, No. 1, 5-37, http://dx.doi.org/ 10.1680/geot.2011.61.1.5.
Duncan, J. M. (2000). Factors of safety and reliability in geotechnical engineering. J. Geotech. Geoenviron. Engng 126, No. 4, 307-316.

Fenton, G. A. (1999). Estimation for stochastic soil models. J. Geotech. Geoenviron. Engng 125, No. 6, 470-485.

Fenton, G. A. \& Griffiths, D. V. (2008). Risk assessment in geotechnical engineering. New York, NY, USA: John Wiley \& Sons.

Fenton, G. A. \& Vanmarcke, E. H. (1990). Simulation of random fields via local average subdivision. J. Eng. Mech. 116, No. 8, 1733-1749.

Griffiths, D. V. \& Fenton, G. A. (2009). Probabilistic settlement analysis by stochastic and random finite element methods. J. Geotech. Geoenviron. Engng 135, No. 11, 1629-1637.

Griffiths, D. V., Paiboon, J., Huang, J. \& Fenton, G. A. (2008) Numerical analysis of beams on random elastic foundations. Proc. 9th Int. Congr. on Numerical Methods in Engineering and Scientific Applications (CIMENICS '08), Isla de Margarita, Venezuela, CI 19-25.

Haldar, S. \& Basu, D. (2011). Beam on spatially random elastic foundation. Proc. GeoRisk 2011, Atlanta, GA (CD-ROM).

Hetenyi, M. (1946). Beams on elastic foundations. Ann Arbor, MI, USA: University of Michigan Press.

Lee, I. K., White, W. \& Ingles, O. G. (1983). Geotechnical engineering. London, UK: Pitman.

Paiboon, J. (2008). Probabilistic analysis of laterally loaded piles. Master's thesis, Division of Engineering, Colorado School of Mines, Golden, CO, USA.

Phoon, K. K. \& Kulhawy, F. H. (1995). On quantifying inherent soil variability. In Uncertainty in the geologic environment: From theory to practice (eds C. D. Shackelford, P. P. Nelson and M. J. S. Roth), Geotechnical Special Publication No. 58, pp. 326-340. Reston, VA, USA: ASCE.

Poulos, H. G. (1989). Pile behaviour-theory and application. Géotechnique 39, No. 3, 365-415, http://dx.doi.org/10.1680/ geot.1989.39.3.365.

Poulos, H. G. \& Davis, E. H. (1974). Elastic solutions for soil and rock mechanics. New York, NY, USA: John Wiley \& Sons.

Poulos, H. G. \& Davis, E. H. (1980). Pile foundation analysis and design. New York, NY, USA: John Wiley \& Sons.

Prakash, S. \& Sharma, H. D. (1990). Pile foundations in engineering practice. New York, NY, USA: John Wiley \& Sons.

Ramu, S. A. \& Ganesar, R. (1995). Response and stability of a stochastic beam-column using stochastic FEM. Comput. Struct 54, No. 2, 207-221.

Randolph, M. F. (1981). The response of flexible piles to lateral loading. Géotechnique 31, No. 2, 247-259, http://dx.doi.org/ 10.1680/geot.1981.31.2.247.

Reese, L. C. \& Van Impe, W. F. (2001). Single piles and pile groups under lateral loading. Rotterdam, the Netherlands: A. A. Balkema.

Smith, I. M. \& Griffiths, D. V. (2004). Programming the finite element method, 4th edn. Chichester, UK: John Wiley \& Sons.

Vanmarcke, E. H. (1984). Random fields: Analysis and synthesis. Cambridge, MA, USA: MIT Press

Zhang, J. \& Ellingwood, B. (1995). Effects of uncertain material properties on structural stability. J. Struct. Engng 121, No. 4, $705-716$. 